\title{
REMARK ON THE CHAIN RULE OF FRACTIONAL DERIVATIVE IN THE SOBOLEV FRAMEWORK
}

\author{
KAZUMASA FUJIWARA
}

Abstract. A chain rule for power product is studied with fractional differential operators in the framework of Sobolev spaces. The fractional differential operators are defined by the Fourier multipliers. The chain rule is considered newly in the case where the order of differential operators is between one and two. The study is based on the analogy of the classical chain rule or Leibniz rule.

Mathematics subject classification (2020): 46E35, 42B25.

Keywords and phrases: Chain rule, power product, fractional derivative, Sobolev spaces.

\section{REFERENCES}

[1] J. BeRGH AND J. LÖFSTRÖM, Interpolation spaces, Springer, Berlin/Heiderberg/New York, 1976.

[2] M. F. Christ AND M. I. Weinstein, Dispersion of small amplitude solutions of the generalized Korteweg-de Vries equation, J. Funct. Anal., 100, 1, (1991), 87-109.

[3] K. Fujiwara, M. IKeda, AND Y. WAKASUgi, The Cauchy problem of the semilinear second order evolution equation with fractional Laplacian and damping, to appear in Nonlinear Differ. Equ. Appl.

[4] K. Fujiwara, V. Georgiev, And T. Ozawa, Higher order fractional Leibniz rule, J. Fourier Anal. Appl., 24, (2018), 650-665.

[5] J. Ginibre, T. OZAWA, AND G. Velo, On the existence of the wave operators for a class of nonlinear Schrödinger equations Annales de l’I. H. P., Physique théorique, 60, 2, (1994), 211-239.

[6] L. Grafakos, Classical Fourier analysis, Springer, New York, 2008.

[7] L. Grafakos And S. OH, The Kato-Ponce Inequality, Comm. Partial Differential Equations, 39, 6, (2014), 1128-1157.

[8] H. Hajaiej, L. Molinet, T. Ozawa, And B. Wang, Necessary and sufficient conditions for the fractional Gagliardo-Nirenberg inequalities and applications to Navier-Stokes and generalized boson equations. Harmonic analysis and nonlinear partial differential equations, RIMS Kokyuroku Bessatsu, B26, (2011), 159-175.

[9] T. Kato And G. Ponce, Commutator estimates and the Euler and Navier-Stokes equations, Comm. Pure Appl. Math., 41, 71, (1988), 891-907.

[10] E. LenZMANn AND A. SCHIKORRA, Sharp commutator estimates via harmonic extensions, Nonlinear Analysis, 193, (2020), 111375.

[11] M. NAKAMURA AND T. OZAWA, The Cauchy problem for nonlinear wave equations in the homogeneous Sobolev space, Ann. Inst. H. Poincaré Phys. Théor, 71, (1999), 1, 199-215. 